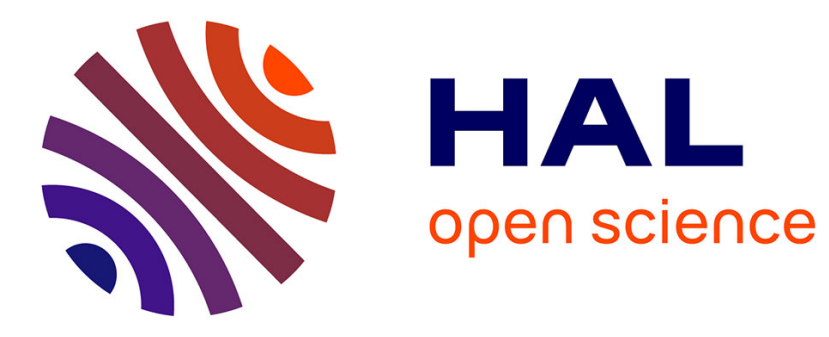

\title{
Transmission Electron Microscopy of Metallic Multilayers
}

\author{
M. Walls, J.-P. Chevalier, Martin Hÿtch
}

\section{To cite this version:}

M. Walls, J.-P. Chevalier, Martin Hÿtch. Transmission Electron Microscopy of Metallic Multilayers. Journal de Physique IV Proceedings, 1996, 06 (C7), pp.C7-213-C7-223. 10.1051/jp4:1996728 . jpa00254516

\section{HAL Id: jpa-00254516 https://hal.science/jpa-00254516}

Submitted on 1 Jan 1996

HAL is a multi-disciplinary open access archive for the deposit and dissemination of scientific research documents, whether they are published or not. The documents may come from teaching and research institutions in France or abroad, or from public or private research centers.
L'archive ouverte pluridisciplinaire HAL, est destinée au dépôt et à la diffusion de documents scientifiques de niveau recherche, publiés ou non, émanant des établissements d'enseignement et de recherche français ou étrangers, des laboratoires publics ou privés. 


\title{
Transmission Electron Microscopy of Metallic Multilayers
}

\author{
M.G. Walls, J.-P. Chevalier and M.J. Hÿtch \\ Centre d'Etudes de Chimie Métallurgique, CNRS, 15 Rue G. Urbain, \\ 94407 Vitry sur Seine cedex, France
}

\begin{abstract}
Resume: We give an overview of use of the transmission electron microscope (TEM) in the characterisation of metallic multilayers. The different types of structural information available from phase and diffraction contrast imaging, as well as the various diffraction modes, are described. The particular usefulness of techniques such as the Fresnel fringe method for multilayer interface characterisation is emphasised. The use of analytical TEM and scanning TEM (STEM) for chemical characterisation is also covered. Special considerations and methods applying to the study of magnetic materials are briefly considered. All points covered are illustrated with examples from the recent literature.
\end{abstract}

\section{INTRODUCTION}

The unique properties of metallic multilayers (MMs) are of interest, both from the fundamental point of view and because of their potential applications. Naturally, the fulfillment of this potential requires a thorough understanding of the fundamental processes at work - a fact which is reflected by the considerable research effort devoted to MMs at present. In this paper we will describe the contribution of transmission electron microscopy (TEM) to the understanding of these systems. The kind of information that can be obtained in the microscope will be described along with its use in the more general study of multilayers. In an earlier review [1] the principles of image formation.and the relations between image contrast and sample structure were covered in some detail. Here we will concentrate on the most recent applications and cover some topics, such as magnetic domain imaging, which were not included in [1].

Of particular interest in MMs are the magnetic properties (giant magnetoresistance etc.), the optical characteristics (X-ray mirrors), the electronic structure, and more generally, the growth mechanisms, the mechanical properties and stability. The microscope can, in certain circumstances, furnish information about the first three of these questions. However, with regard to all these properties (and practically all other physical properties of solids) it is useful to have a detailed knowledge of the microstructure. In particular, of crucial importance are the grain size and orientation (texture), layer regularity (thickness, uniformity etc.) and the quality of the interfaces. The TEM is ideally suited to the study of these aspects of the multilayer. It is in combining the results of TEM and other microscopic techniques with measurements of "macroscopic" properties, that the relations between microstructure and behaviour (optical, magnetic, etc.) can be established. We will begin with a brief description of the TEM and the various analytical signals that can be used for these studies.

\section{THE MICROSCOPE AND THE SAMPLE}

\subsection{The microscope}

The TEM consists essentially of an evacuated column with an electron source at the top, followed by beamforming lenses which direct the electrons onto the surface of the sample in the form of a parallel beam or a focussed probe. The sample itself is a thin film through which the electrons pass. The objective and subsequent lenses form the magnified image, or diffraction pattern, which can be observed and recorded. 
Figure 1 is a simplified schema of the microscope, showing the interactions of interest here. Elastic scattering, or diffraction, is of great value for structure determinations via diffraction patterns and for diffraction contrast imaging of the microstructure. Diffracted electron waves may also be recombined with the non-scattered beam to produce a high resolution or lattice image. Images of magnetic domains can be formed using electrons scattered through very small angles by the domain magnetic field. Fresnel fringe imaging, at large defoci, is also useful in this context and for layer quality and interface studies. The inelastic scattering is related to processes of electron excitation in the sample, and the electron energy-loss spectrum (EELS) contains chemical and electronic information. In addition, the sample emits X-rays of energies characteristic of the elements present which can be used for chemical analysis. Many other signals are present but we will consider only those mentioned above, since they are the ones currently employed in MM research.

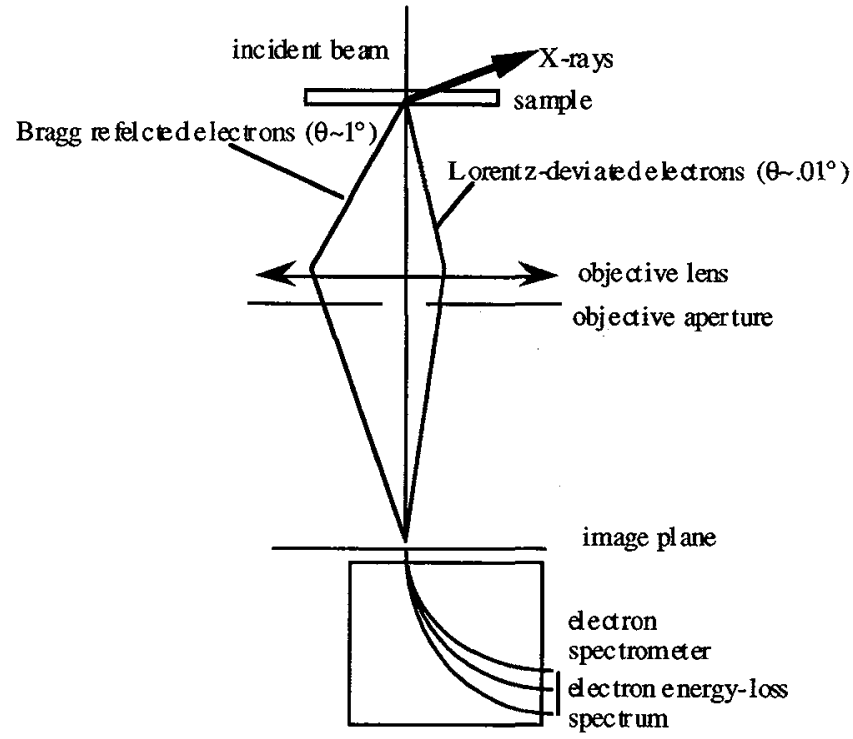

Figure 1: beam-sample interactions in the TEM (simplified)

\subsection{The sample}

A good sample is the first pre-requisite for high quality TEM experiments. Ideally, the specimen will be thin over as large an area as possible ("thin" means $\sim 10 \mathrm{~nm}$ for high resolution work, and $\leq 100 \mathrm{~nm}$ for most other purposes). It will also be clean, without too much amorphous material on the surfaces, and will be representative of the bulk material.

The first condition can be fulfilled with practice using one of a range of preparation techniques depending on the nature of the sample and the orientation required. In general, for plan view samples (viewing direction perpendicular to the MM interfaces) standard chemical or electrochemical thinning techniques can be applied, although some workers prefer to finish with ion-beams. For cross-sectional samples "sandwich" methods are often employed, in which two pieces of the multilayer and its support are glued face to face (MMs on the inside) and mechanical thinning is carried out in the direction parallel to the interface plane until the thickness is $50 \mu \mathrm{m}$ or less. Ion-beam thinning is then used to perforate the sample. An amorphous layer is always present at the surfaces of samples prepared by these methods. Its thickness can be reduced by finishing the ion-beam thinning at very low angles and voltages, but it cannot be eliminated entirely, see for example [2]. An alternative technique is the recently developed and entirely mechanical "tripod" method [3] in which increasingly fine polishing media are used to produce a low-angle wedge of material, and appears in most cases to be essentially free of amorphous overlayers and dirt. However, this technique can be quite difficult to apply to heterogeneous materials in cross-section and its use is so far limited in MM studies.

Other techniques include cleavage and crushing when the support is a brittle material (these yield clean samples but in general the amount of thin area is limited) and ultramicrotomy, which yields larger thin areas, but in general requires that the substrate be removed first, which is often impossible.

The problem of the thin sample being representative of the bulk material is more difficult. Strained-layer superlattices, in which a lattice mismatch between the materials in the multilayer creates in-built strains in 
the material, pose a particular problem, since these strains are relaxed to a large extent in the thin material. This is not a particularly common difficulty in MM studies but one should be aware of the possibility of this and other thin-film effects when interpreting microscope observations.

The choice of sample orientation depends on the type of information sought. It is important to realise that one sample will not usually be sufficient to characterise the material completely. Plan view samples often give a better impression of the overall microstructure of the layers, simply because the relevant thin area is usually much greater. They are also of interest for imaging the interfacial dislocation distribution in epitaxial systems and for texture determinations. However, for an examination of the interface structure and quality, and layer thicknesses and uniformity, a cross-section sample is required. In some cases it may even be necessary to examine cross-sections in more than one azimuthal direction. Fig. 2 shows an example of two images of an $\mathrm{Fe} / \mathrm{Cr}$ multilayer looking along the [001] and [110] directions [4]. Clearly, if only the first sample had been available, the saw-tooth structure seen in the second image would not have been detected.

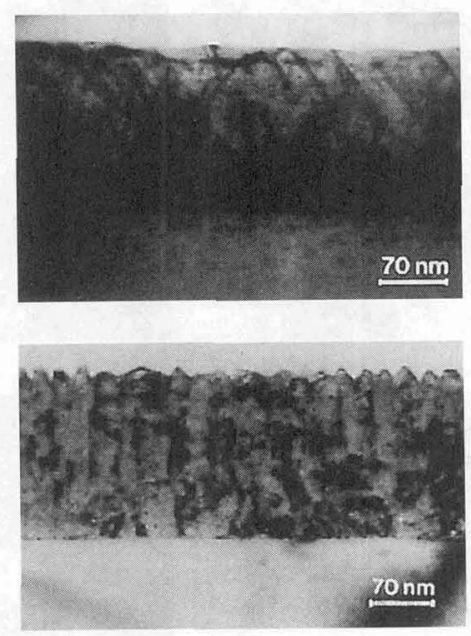

Figure 2: Bright field TEM images of a Fe/Cr(110) superlattice; (a) cross section along the [001] direction; (b) cross section along the [110] direction. (Courtesy of Folkerts and Hakkens [4]).

\section{INFORMATION FROM THE DIFFRACTION PATTERN}

The TEM can furnish a diffraction pattern from a selected part of the specimen. The Bragg angles are small for the short wavelength electrons used, and the accuracy of measurements is inferior to that of X-ray diffraction methods. However, the TEM has the advantage of high spatial resolution: the pattern can be acquired from small specific areas of the sample, which makes it possible to study inhomogeneities.

Here, we will not give the details of diffraction pattern formation, which can be found in numerous other texts [eg. 5,6]. We will just resume the principal types of patterns usually obtained from MMs.

In general, standard "selected area" electron diffraction (SAED) is employed, in which a small region of the sample is selected by an aperture and illuminated with a parallel beam. Amorphous material has an SAED pattern consisting of diffuse rings. Small crystals in random orientations give sharper but still continuous rings. If there is texture present (preferred orientation for the crystals) this can be revealed by discontinuities in the rings, corresponding to those azimuthal angles (with respect to the incident beam direction) at which few or no crystals are oriented (the intensities of the parts of the ring corresponding to the preferred orientations are of course enhanced). Regions consisting of larger crystals (of which fewer are included by the aperture) give discontinuous rings consisting of discrete spots. In addition, in cubic systems, the relative ring radii have well known sequences which yield an immediate distinction between for example face centred cubic and body centred cubic structures. A very complete study, illustrating the use of these effects in the study of Ni-Ag multilayers has been given by Wang and Simon [7]. One example showing texture and grain-size effects after different annealing times is shown in fig. 3. 

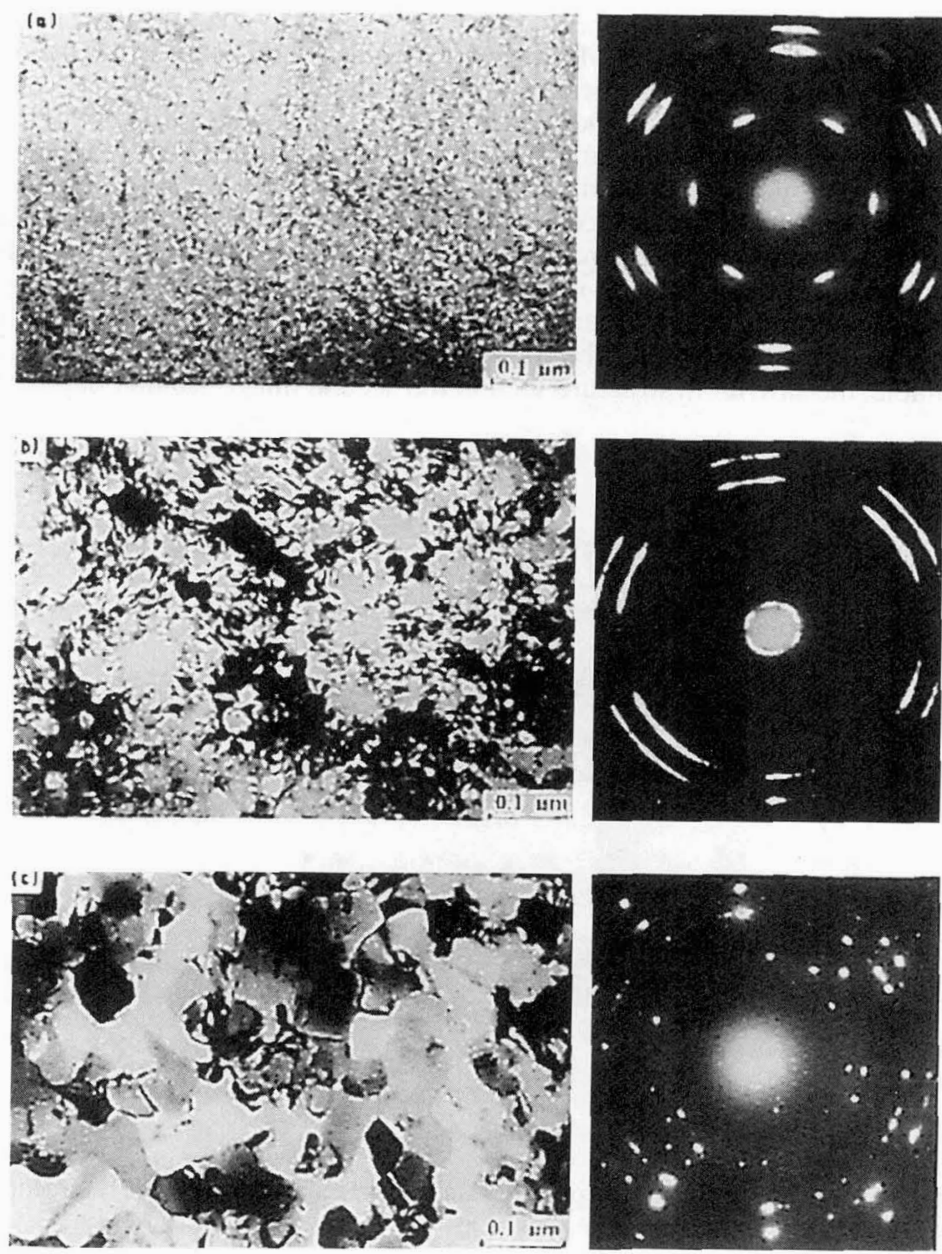

Figure 3: TEM bright field images and electron diffraction panterns of $5 / 5$ samples showing the effects of in situ annealing; (a) as-deposited state; (b) $300^{\circ} \mathrm{C}, 30 \mathrm{~min}$; (c) $450^{\circ} \mathrm{C}, 30 \mathrm{~min}$. (Coutesy of Wang and Simon [7].)

A further use of the diffraction pattern of specific interest here is in the measurement of the multilayer period. All periodic structures in the sample give rise to diffraction spots whose spacing is inversely proportional to the period. Thus the principal spots corresponding to the crystal Bragg reflections have satellite spots on either side whose spacing gives the multilayer period. The spacing of the atomic plane Bragg reflections gives an internal calibration of the pattern. An example from the Si-Mo system [8] is shown in fig. 4.

Up till now, there appears to have been little use made of the other diffraction modes in the microscope (convergent beam etc) in the study of MMs. The paper mentioned above [8] was in fact mainly concerned with nano-diffraction using a fine probe placed at $\mathrm{Si}$-Mo interfaces. It was shown that there is streaking towards the Mo layer in the non-diffracted spot, and this is explained by electron refraction in the Mo which has a higher inner potential than the $\mathrm{Si}$ (see below - Fresnel imaging). Furthermore, images acquired using the streaked part of the spot show the interface regions and reveal a difference in sharpness between the interfaces made by Si deposition on Mo and by Mo deposition on Si. 

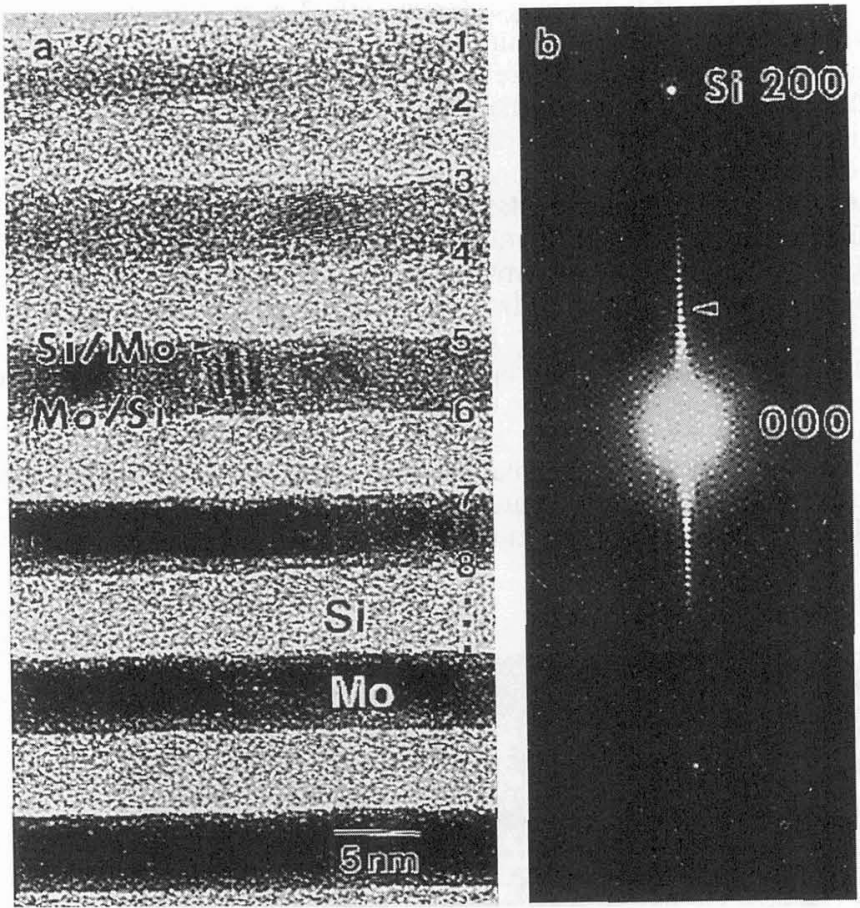

Figure 4: High resolution image of Mo/Si multilayer (a) with denoted Si/Mo (odd numbers) and Mo/Si (even numbers) intefaces. SAD pattern from multilayer (b) in which spots due to bilayer periodicity (arrowed) obscure streaking from refraction at interfaces. (Courtesy of Gajdardziska et al. [8].)

\section{IMAGING OF MULTILAYERS USING CONVENTIONAL METHODS}

\subsection{Diffraction contrast}

Diffraction contrast in an image is caused by the diffraction of electrons out of (or into) the objective lens aperture. It again reveals details about the microstructure at medium resolution and is used in conjunction with SAED. Contrast between grains oriented differently is generally strong (different amounts of Bragg scattering) and thus plan view images at fairly low magnification give a quick measure of grain sizes (fig. 3). The shape of the grains is also sometimes of importance, columnar growth perpendicular to the layers being fairly common and cross-sectional diffraction contrast imaging shows this particularly clearly [9]. Typically then, the microscope is used first to characterise the material at the microstructural level using SAED and diffraction contrast, then the details at the interfaces are probed by higher resolution imaging as described below.

\subsection{High resolution microscopy}

Using modern microscopes, images can readily be obtained with a spatial resolution of about $2 \AA$. This is sufficient to visualise the crystal structure when looking along the principal axes of most metallic crystals. To a first approximation, for very thin crystals consisting of light elements, such images represent a projection of the crystal potential in the incident beam direction, atomic columns appearing dark and open channels in the structure appearing lighter. However, for most real specimens this approximation is not 
directly applicable, and reversals of the contrast occur with thickness and/or defocus variations. Further complicated effects will occur at interfaces, grain boundaries and defects. Interpretation of the image at the atomic level is thus hazardous and should wherever possible be accompanied by numerical simulations of what the contrast would be expected to be for the structure one believes to be observing. Such simulations, which are routinely included in publications concerning other materials (semiconductor structures for example) are almost entirely absent from the metallic multilayer literature. The conclusions sometimes reached about details of the atomic arrangements at the interface plane, based on intuitive interpretations of the image contrast, are thus of questionable value. A notable exception is the analysis of the apparent position of atomic columns in high resolution images of strained mulitlayers of $\mathrm{Au}-\mathrm{Ni}$ [10]. By combining the experimental images with those obtained from simulations, chemical profiles across the multilayers could be obtained to a high spatial resolution. Detailed studies [11] also using image simulations, have explained the contrast (in fact the lack of contrast) in Pt-Co multilayers. Is was shown that the invisibility of the nominally $3 \AA$ Co layers in the images implies interdiffusion with the Pt to the extent that Co occupation on any plane does not exceed $40 \%$.

High resolution images are very useful for the qualitative characterisation of multilayers and their interfaces, particularly when the two materials concerned are of significantly differing average atomic number $Z$. (If $Z$ is similar on both sides of the interface, the rather weak phase contrast may not be sufficient to reveal

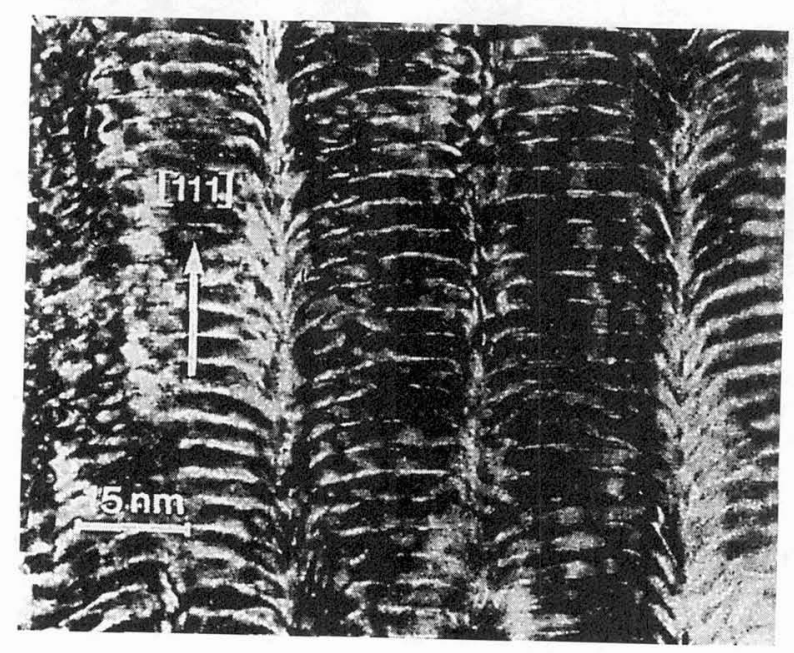

Figure 5: Cross section bright field image of $0.8 \mathrm{~nm} \mathrm{Co} / 2.2 \mathrm{~nm} \mathrm{Pd}$ multilayer, $\mathrm{T}_{\mathrm{S}}=20^{\circ} \mathrm{C}$. (Courtesy of F. Hakkens [23].)

clearly the interface plane.) A considerable amount of literature exists in which high resolution and/or diffraction contrast images are presented, comparing the microstructure for a few samples varying somewhat in composition or preparation methods both for magnetic type [12-17] and X-ray optical [18-22] applications. An example of a particularly interesting and complete study is that of Hakkens et al. [23] in which for Co-Au MMs, the deposition conditions are shown to have a marked effect on the layer structure. Pronounced curvature of the layers within a columnar grain is observed for UHV vapour deposited multilayers and magnetron sputtered films deposited at high argon pressures (fig. 5), but is absent at low argon pressures. This would appear to be related to the intrinsic stresses in the films, which are low, leading to loosely packed columns, in the former cases, and high, giving dense column-packing, in the latter.

Perhaps of more interest from the technological point of view is research in which the variations in grain sizes and defect types and densities from one sample to another are correlated with the measurements of other properties of interest for applications, for example giant magnetoresistance (GMR) and other magnetic properties [24-29] or X-ray reflectivity [30]. A good example is the study of Permalloy-Au MMs, in which increasing annealing times lead to increasing interfacial roughness and eventual bridging between the magnetic Permalloy layers, which is in turn correlated with decreasing GMR (fig. 6) [31]. A short review concentrating on high resolution MM studies has been given by Smith et al. [32]. 


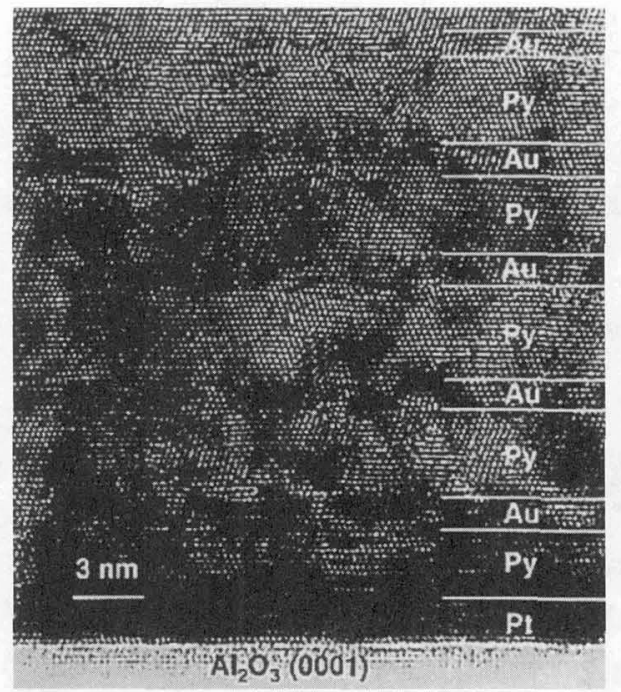

Figure 6: HREM micrograph of annealed Py/Au multilayer showing the decrease in Au layer as a result of Au intermixing with the Py layer. (Courtesy of N. Thangaraj [31].)

\section{SPECIAL IMAGING TECHNIQUES AND ANALYTICAL MICROSCOPY}

\subsection{The use of Fresnel fringes to measure layer and interface quality}

A MM whose layers are aligned parallel to the incident beam yields, at large defoci, an image exhibiting Fresnel fringes at the interfaces between one metal and the other. This contrast is frequently exploited to visualise the interface in cases where conventional types of contrast are weak (eg. for metals of similar Z). For example, Valet et al. [33] use it to show the deterioration in layer regularity as a function of distance form the substrate in $\mathrm{Ni}_{80} \mathrm{Fe}_{20} / \mathrm{Cu} / \mathrm{Co}$ MMs. The fringes, which are due to the difference in mean inner potential between the two materials, can also be used to characterise the interface itself. For a given pair of metals, the fringe spacings and intensities are functions of defocus, sample thickness and interface abruptness. A through-focal series of images from the sample is thus compared with simulated Fresnel fringe profile series assuming different interface widths and the width value giving the best fit is assumed to correspond to the real interface width. The technique has been successfully applied to $\mathrm{Cu}$-Co multilayers prepared by electrochemical methods [34]. Figure 7 shows one of the experimental images analysed. It is also possible using this method [34] to separate the effects of interface roughness from those due to interdiffusion, i.e. to distinguish an abrupt but non-flat interface from one whose plane is flat but where some interdiffusion of the metal has occured from one side to the other.

\subsection{Special techniques for magnetic domain imaging}

One of the main applications envisaged for MMs is as the active components in magnetic sensors. Research in this area will require techniques which can probe the local magnetic properties of the multilayers. The electron microscope is well adapted to the problem, since electrons interact with magnetic fields. The trajectory of an electron of velocity $\mathbf{v}$ traversing a magnetised sample is deviated by the Lorentz force in the direction $\mathbf{v} \times \mathbf{B}$ where $\mathbf{B}$ is the magnetic induction in the sample. Thus, two domains magnetised in opposite directions will deflect the beam in opposite directions, leading to a splitting of the diffraction spots. In general the deflection is very small - very much less than a Bragg angle but it can be used to give contrast in the image. The use of the effect is known as Lorentz microscopy and has two principal variants. In the first of these, known as Foucault microscopy, the objective aperture is placed so as to include only one of the split spots in the back focal plane. Domains contributing to this spot thus appear 


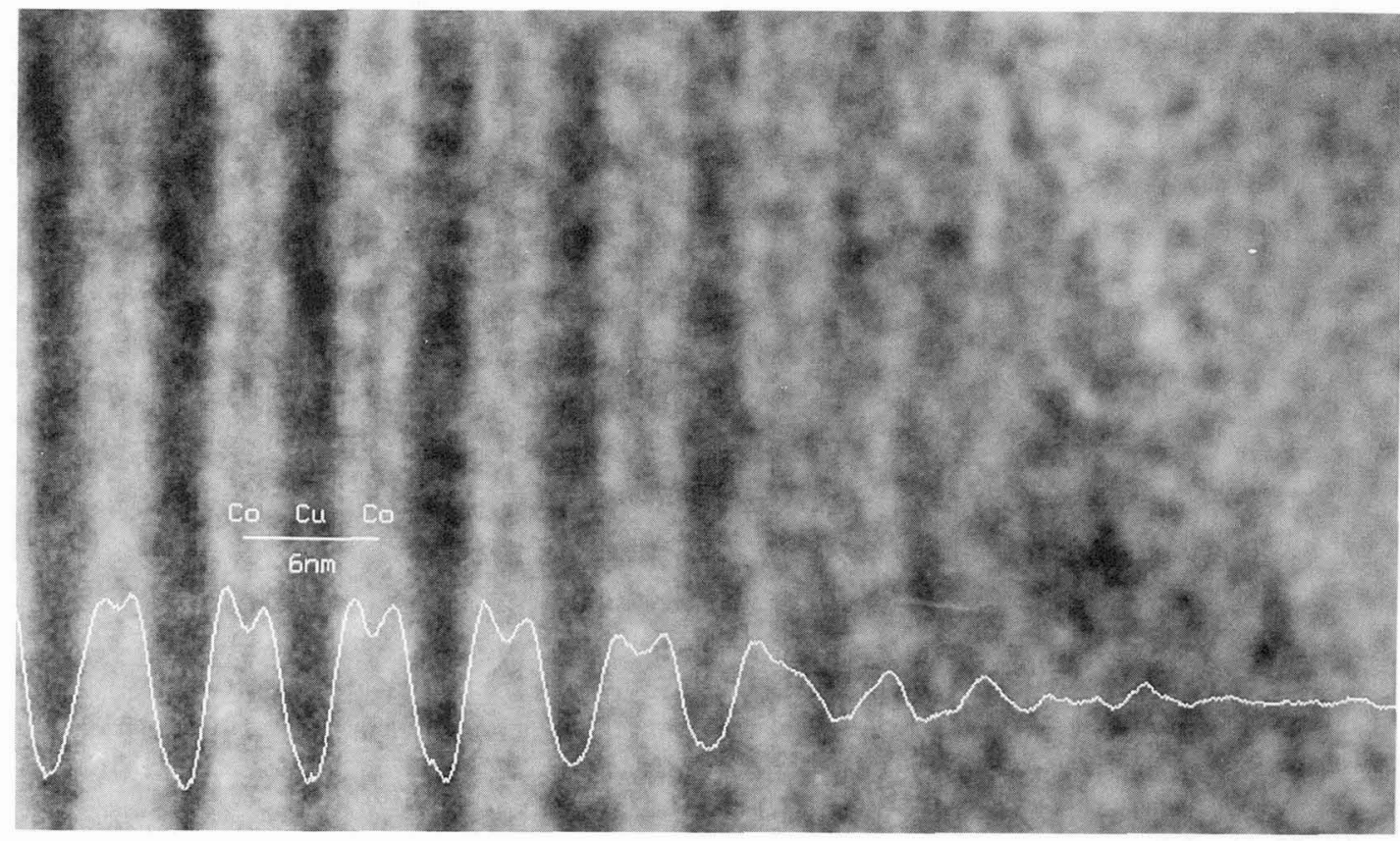

Figure 7: TEM image of Co-Cu multilayer taken at a defocus of $720 \mathrm{~nm}$ showing Fresnel fringe contrast. The intensity profile after averaging along the layers is also shown.

bright and those contributing to the excluded spot appear dark. The second contrast mechanism is a Fresnel effect similar to that described above, but due here to the Lorentz force. In a defocussed image the domain walls appear as bright or dark lines, depending on whether the electrons are deflected towards or away from the wall.

In practice, the application of either technique requires rather special operating conditions and is best performed in a dedicated instrument. Long focal length objective lenses whose field does not saturate the sample, and high precision small objective apertures which can be placed exactly in the objective back focal plane are desirable modifications [35]. An example from Kim et al.[36] showing the two types of contrast in the same region of a $\mathrm{Co}-\mathrm{Cu} \mathrm{MM}$ is shown in fig. 8. This is a rather complete study, showing the relation between microstructure, magnetic domain structure and GMR. For example, in the sample with the highest GMR, domain contrast is weak, and in that with the lowest it is strong, which agrees with the hypothesis of antiferromagnetic coupling being associated with high GMR and ferromagnetic coupling with low GMR. Other recent studies using these methods include Foucault images from Cr-Fe films [37] and Fresnel imaging of (Co-NiFe-Co)-Cu structures [38].

An alternative approach is to use the scanning TEM (STEM) geometry, in which the image is acquired sequentially by scanning a focussed probe across the sample and simultaneously measuring the transmitted intensity. If the transmitted beam detector is split into quadrants, small deflections will be registered as variations in the relative intensities reaching each quadrant. Images created using differences in the quadrant signals can be used to give a map of the magnetisation vector. This is known as the differential phase contrast (DPC) method [39] and has been successfully applied to a number of multilayer problems, for example domain size and wall structure determinations in Co-Pt multilayers $[39,40]$

\subsection{Analytical methods}

The term "analytical microscopy" refers to the use of two principal techniques. Energy dispersive X-ray (EDX) analysis uses the intensity of characteristic X-rays emitted by the elements under the beam to quantify the concentrations of those elements. Electron energy-loss spectroscopy (EELS) as its name implies uses the characteristic energy losses in the spectrum of the transmitted electrons to obtain the same information. Both techniques thus furnish a chemical analysis on the microscopic scale. These methods 


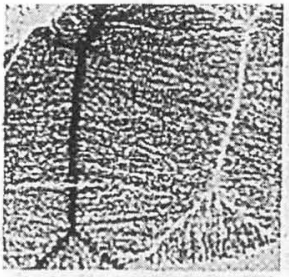

(a)

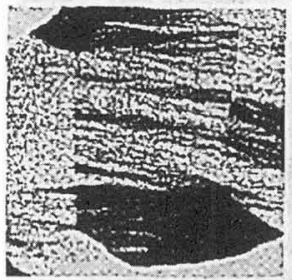

(c)

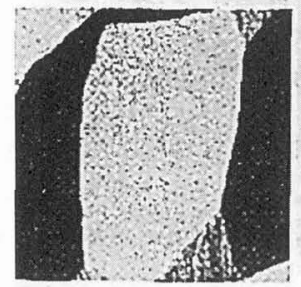

(e)

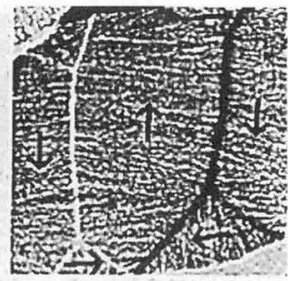

$\therefore(b)$

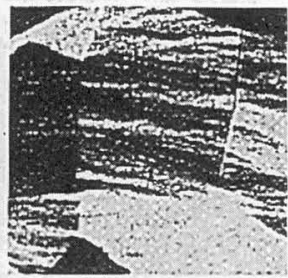

(d)

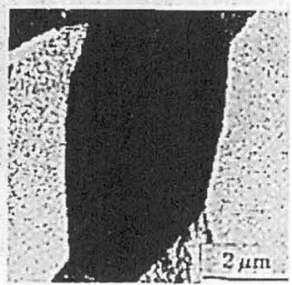

(i)

Figure 8: Lorentz micrographs showing the magnetic domain structure of a $(2 \mathrm{~nm} \mathrm{Co} / 0.5 \mathrm{~nm} \mathrm{Cu})_{40} \mathrm{sample}$. Two complementary Fresnel images are shown in (a) and (b), and four Foucault images in (c)-(f). The direction of the displacement of the objective aperture was (d) at $180^{\circ}$, (e) $90^{\circ}$, and (f) $270^{\circ}$ relative to (c). The magnetization directions are shown in (b). (Courtesy of J. D. Kim [36]).

would seem well adapted to measuring concentration profiles of particular elements across the interfaces in a multilayer, particularly EELS, which can have a spatial resolution of the order of the minimum probe size in the microscope, which can be under $1 \mathrm{~nm}$ in an instrument with a field emission source. The literature on the subject is however as yet not particularly abundant. This may be due to the problems which often beset the use of these methods at the upper limits of resolution, principally, severe carbon contamination of the area under the beam, rendering analysis impossible if the sample is not extremely clean. Nevertheless some useful studies have been performed using these techniques. Examples include EELS measurements of elemental concentration profiles with nanometer resolution in $\mathrm{Fe}-\mathrm{Co}$ and $\mathrm{Au}-\mathrm{Ni} \mathrm{MMs}$ [41], and a comparison of EELS and EDX measurements of impurity concentrations ( $\mathrm{Ti}$ in $\mathrm{Ni}$ and $\mathrm{Ni}$ in $\mathrm{Ti}$ ) in Ni-Ti multilayers, showing that the inferior spatial resolution in EDX leads to an overestimation of the impurity levels [42].

An alternative approach is to use energy filtered imaging, with parallel illumination rather than a probe. If images are acquired at energy-loss values just before and after the threshold for a given element a map of the distribution of that element can be constructed by subtracting the former from the latter. More sophisticated methods should be used for a quantitative map, but as a first approximation this approach can be quite useful, and has been successfully applied to map $\mathrm{Cr}$ in NiFe-Cr [43] and Al and Ti in Al-Ti [44] MMS with sub-nanometer resolution. 

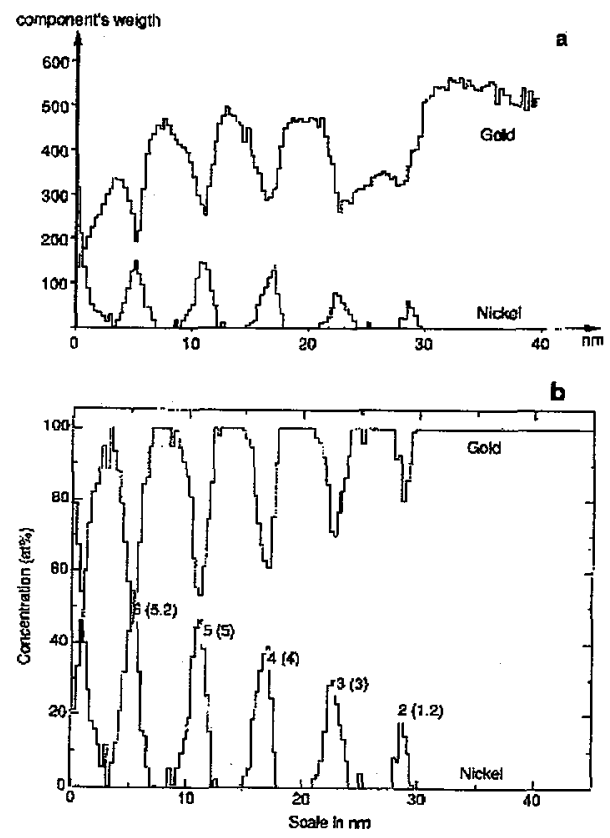

Figure 9: Spacial dependence of weight of $\mathrm{Ni}$ and Au components across mutilayer, extracted from processing of line spectrum (a). Same data displayed in terms of relative atomic concentrations (b). (Courtesy of M. Tencé. [41].)

\section{CONCLUSIONS}

TEM can be applied to the study of metallic multilayers in a variety of ways. The particular advantage of the technique is the wide variety of information, structural, chemical, and magnetic, available at the high spatial resolution which is required to characterise these nanometer-scale structures. A more rigorous and increasingly a quantitative approach to the interpretation of images and diffraction data will improve still further the quality of information obtainable.

\section{References}

[1] Chevalier J.P., in Metallic Multilayers Materials Science Forum 59-60 eds. Chamerod and Hillairet. Trans Tech Publications Zurich (1989) 141

[2] Sheng T. T. and Marcus R. B., J. Electrochem. Soc. 127 (1980) 737-743.

[3] Benedict J., Anderson R., Klepeis S. and Choker M., Mater. Res. Soc. Symp. Proc. 199 (1990) $189-190$.

[4] Folkerts W. and Hakkens F., J Appl Phys 73, (1993) 3922-3925.

[5] Spence J. C.H., Experimental High Resolution Electron Microscopy, Oxford University Press (1988)

[6] Reimer L. Transmission Electron Microscopy, Physics of Image Formation and Microanalysis, Spriger Ser. Opt. Sci. 36 Springer, Berlin, Heidelbereg (1984)

[7] Wang Y. S. and Simon J. P., J Mater Sci 27 (1992) 6755-6764.

[8] Gajdardziska-Josifovska M., Weiss J. K. and Cowley J. M., Ultramicroscopy 58 (1995) 65-78.

[9] Visokay M. R., Kuwabara M. and Hayashi H., J Magn Magn Mater 126 (1993) 131-140.

[10] Bayle P., Deutsch T., Gilles B., Lançon F., Marty A. and Thibault J., Ultramicroscopy 56 (1994) 94-107.

[11] Cho N. H., Krishnan K. M., Lucas C. A. and Farrow R. F. C., J Appl Phys 72 (1992) 5799--5807.

[12] Modak A. R., Parkin S. S. P. and Smith D. J., Ultramicroscopy 47 (1992) 375-382.

[13] Modak A. R., Parkin S. S. P. and Smith D. J., J Magn Magn Mater 129 (1994) 415-422.

[14] Bertero G. A., Hufnagel T. C., Clemens B. M. and Sinclair R., J Mater Res 8 (1993) 771-774.

[15] Dohnomae H., Jpn J Appl Phys Pt 133 (1994) 1499-1508. 
[16] Wang L., Fricoteaux P., Yuzhang K., Troyon M., Bonhomme P., Douglade J. and Metrot A., Thin Solid Films 261 (1995) 160-167.

[17] Snoeck E., Sinclair R., Parker M. A., Hylton T. L., Coffey K. R., Howard J. K., Lessmann A. and Bienenstock A. I., J Magn Magn Mater 151 (1995) 24-32.

[18] Jiang S. S., Zou J.,. Cockayne D. J. H, Sikorski A., Hu A. and Peng R. W., Phil Mag B 66 (1992) 229-237.

[19] Jiang S. S., Zou J., Cockayne D. J. H., Sikorski A., Hu A. and Peng R. W., Phys Status Solidi AAppl Res 130 (1992) 373-381.

[20] Ahuja R. and Fraser H. L., J Electron Mater 23 (1994) 1027-1034.

[21] Krawietz R., Wehner B., Meyer D., Richter K., Mai H., Dietsch R., Hopfe S., Scholz R. and Pompe W., Fresenius J Anal Chem 353 (1995) 246-250.

[22] Fullerton E. E., Pearson J., Sowers C. H., Bader S. D., Wu X. Z. and Sinha S. K., Phys Rev BCondensed Matter 48 (1993) 17432-17444.

[23] Hakkens F. J. G., De Veirman A. E. M., Coene W. and Den Broeder F. J. A., J. Mater. Res, 8 (1993) 1019-1027.

[24] Modak A. R., Smith D. J. and Parkin S. S. P., Phys Rev B-Condensed Matter 50 (1994) $4232-4235$.

[25] Bernardi J., Hutten A., Friedrichs S., Echer C. E. and Thomas G., Phys Status Solidi A-Appl Res 147 (1995) 165-175.

[26] Carcia P. F., Li Z. G., Reilly M. and Zeper W. B., J Appl Phys 73 (1993) 6424-6426.

[27] Smith D. J., Li Z. G., Modak A. R., Parkin S. S. P., Farrow R. F. C. and Marks R. F., Scr Metall Mater 30 (1994) 689-694.

[28] Mitwalsky A., Sporl K. and Weller D., J Appl Phys 73 (1993) 4942-4950.

[29] Parker M. A., Hylton T. L., Coffey K. R. and. Howard J. K, J Appl Phys 75 (1994) 6382-6384.

[30] Takenaka H., Kawamura T., Ishii Y. and Asagiri S., J Appl Phys 78 (1995) 5227-5230.

[31] Thangaraj N. and Krishnan K. M. and Farrow R. F. C., Scripta Metall. 33 (1995) 1667-1677.

[32] Smith D. J., Li Z. G., Modak A. R., Parkin S. S. P., Farrow R. F. C. and Marks R. F. Scripta Metall. 30 689-694

[33] Valet T., Galtier P., Jacquet J. C., Meny C. and Panissod P., J Magn Magn Mater 121 (1993) $402-405$.

[34] Nallet P., Chassaing E., Walls M. G. and Hÿtch M. J., J. Appl. Phys. 79 (1996) 6884-6889

[35] Doole R. C., Petfordlong A. K. and Jakubovics J. P., Rev Sci Instr 64 (1993) 1038-1043.

[36] Kim J. D., Petfordlong A. K., Jakubovics J. P., Evetts J. E. and Somekh R., J Appl Phys 76 (1994) 2387-2394.

[37] Gu E., Daboo C., Bland J. A. C., Gester M., Ives A. J. R., Brown L. M., Stelmashenko N. A. and Chapman J. N., J Magn Magn Mater 126 (1993) 180-184.

[38] Heyderman L. J., Chapman J. N. and Parkin S. S. P., J Magn Magn Mater 138 (1994) 344-354.

[39] Donnet D. M., Chapman J. N., Vankesteren H. W. and Zeper W. B., J Magn Magn Mater 115 (1992) 342-352.

[40] Ploessl R., Chapman J. N., Scheinfein M. R., Blue J. L., Mansuripur M. and Hoffmann H., J Appl Phys 74 (1993) 7431-7437.

[41] Tencé M., Quartuccio M. and Colliex C., Ultramicroscopy 58 (1995) 42-54.

[42] Stadelmann P., Leifer K. and Verdon C., Ultramicroscopy 58 (1995) 35-41.

[43] Kimoto K., Hirano T., Usami K. and Hoshiya H., Jpn J Appl Phys Pt 233 (1994) L1642-L1644.

[44] Wang Z. L. and Shapiro A. J., Ultramicroscopy 60 (1995) 115-135. 